Solar Physics

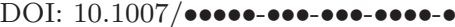

\title{
Particle interactions with single or multiple 3D solar reconnecting current sheets
}

\author{
A. Anastasiadis ${ }^{1}$. C. Gontikakis ${ }^{2}$. \\ C. Efthymiopoulos ${ }^{2}$

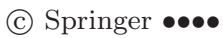

\begin{abstract}
The acceleration of charged particles (electrons and protons) in flaring solar active regions is analyzed by numerical experiments. The acceleration is modelled as a stochastic process taking place by the interaction of the particles with local magnetic reconnection sites via multiple steps. Two types of local reconnecting topologies are studied: the Harris-type and the X-point. A formula for the maximum kinetic energy gain in a Harris-type current sheet, found in a previous work of ours, fits well the numerical data for a single step of the process. A generalization is then given approximating the kinetic energy gain through an X-point. In the case of the multiple step process, in both topologies the particles' kinetic energy distribution is found to acquire a practically invariant form after a small number of steps. This tendency is interpreted theoretically. Other characteristics of the acceleration process are given, such as the mean acceleration time and the pitch angle distributions of the particles.
\end{abstract}

Keywords: Flares, Energetic Particles, Acceleration; Magnetic fields

\section{Introduction}

The study of the energetic particle acceleration process during solar flares still remains an open and challenging problem for solar physics. Solar flares are the manifestation of the energy release process in the solar corona and atmosphere. It is well established that during this energy release process the free magnetic energy is converted, through magnetic reconnection, into heating, bulk motion of the flaring plasma and particle acceleration.

In the framework of the theory of magnetic reconnection process during solar flares, a number of works in the literature have addressed the problem of particle

\footnotetext{
1 National Observatory of Athens, Institute for Space Applications and Remote Sensing GR-15236, Penteli, Greece email: anastasi@space.noa.gr

2 Academy of Athens, Research Center of Astronomy and Applied Mathematics,

Soranou Efessiou 4, GR-11527 Athens, Greece.

email: cgontik@academyofathens.gr

email: cefthim@academyofathens.gr
} 
acceleration. Several authors studied a magnetic field topology with an induced homogeneous electric field, with both fields constant in time, using analytical methods or numerical integration of test particles. The magnetic field topology is either an X-point with or without the presence of a guide field (Bulanov 1980; Deeg, Borovsky and Duric 1991; Bruhwiler and Zweibel 1992; Moses, Finn and Ling 1993; Mori, Sakai and Zhao 1998; Browning and Vekstein 2001; Zharkova and Gordovskyy 2005; Hannah and Fletcher 2006) or a Reconnecting Current Sheet (RCS) (Martens 1988; Martens and Young 1990; Litvinenko 1996; Zharkova and Gordovskyy 2004). The purpose is to study the final kinetic energy distribution of accelerated particles, the condition of adiabaticity of their orbits and the level of charge separation at the ejection points. More realistic steady X-point topologies were derived using the MHD equations (Craig and Litvinenko 2002; Heerikhuisen, Litvinenko and Craig 2002).

In order to model the burst effects many authors incorporate a time dependent electric field in X-points (Hamilton et al. 2003; 2005; Petkaki and MacKinnon 1997; 2007) or adopted a numerical code as Tajima et al. (1987). Wood and Neukirch (2005) adopted an X-point with a nonhomogeneous electric field that was stronger at the center of the X-point. Finally, Kliem (1995) used O-points and X-points combinations to study the effect of the fragmentation of RCS on particles acceleration. On the other hand, several studies (e.g. Benz et al. 1994; Saint-Hilaire and Benz 2002) show that more than $40 \%$ of the released energy in solar flares is deposited to high energetic particles, indicating the close relation between the particle acceleration process and the way (and amount) of the energy released in these highly energetic events. Despite this, a very common approach to building acceleration models (see Miller et al. 1997; Anastasiadis 2002 for reviews) is to consider that the different processes (i.e. energy release, acceleration, transport and radiation) are decoupled. The main reason for such a consideration is the very different temporal and spatial scales on which the different processes evolve. This difference notwithstanding, it should not be neglected that all the processes are interwoven in a way rendering necessary to develop global models for solar flares, i.e., models taking into account the interplay between the individual processes.

Parker (1998) first proposed that the free magnetic energy could be released in the solar corona through multiple dissipation sites. This assumption implies essentially the fragmentation of the energy release in both space and time during solar flares. As several observations, either related to solar flare parameters (e.g. Dennis 1985; Benz 1985; Crosby, Aschwanden and Dennis 1993; Crosby et al. 1998; Aschwanden et al. 1995; 2000) or to emission processes in the solar corona (e.g. Mercier and Trottet 1997; Krucker and Benz 1998), can be interpreted on the basis of such a scenario, several models of particle acceleration were developed that incorporate concepts from the general theory of dynamical systems, such as the complexity of the energy release process. For example, Anastasiadis and Vlahos $(1991,1994)$ assumed the presence of multiple shock waves acting on the particles. Such shocks have the form of smallscale, short-lived magnetic discontinuities inside a flaring active region. Another possibility is the acceleration of particles by multiple DC electric fields (Anastasiadis, Vlahos and Georgoulis 1997; Anastasiadis et al. 2004; Vlahos, Isliker and Lepreti 2004; Dauphin, Vilmer and Anastasiadis 2007). Such fields are associated with the variety of dissipation sites inside an active region. A simulation of the energy release process can then be made by a cellular automaton $(\mathrm{CA})$, the dynamical substrate of which incorporates the assumption that the active region evolves according to the 
dynamical laws of a system being on the status of 'self-organizing criticality' (see Isliker, Anastasiadis and Vlahos 2001 for details on CA models for solar flares).

In the solar corona, large-scale and long lasting current sheets, in which a large number of particles could be accelerated, are unlikely to be present. Recent MHD simulations show that large current sheets are not stable for a long time and are quickly fragmented (Onofri, Isliker and Vlahos 2006). Furthermore, Hughes et al. (2003) have shown that solar flares can be considered as cascades of reconnecting smallscale magnetic loops inside an overall simple large magnetic field topology. In other words, the large-scale magnetic field topology probably determines the location of the magnetic energy release regions only on the scale of the active region. In addition, the complex magnetic environment of an active region together with the turbulent photospheric motions should be taken into account, since these external factors drive the system continuously by adding new stresses to the existing large-scale topologies. In particular, such a type of driving leads necessarily to the formation of multiple reconnecting current sheets (RCS) configurations.

In the present work, we consider the above description as the basis for constructing numerical simulations of the acceleration of particles (electrons and protons) within multiple 3D current sheets developing in an active region. Our basic model is a sequence of encounter events of a population of particles with local current sheets with physical parameters changing stochastically. In order to understand the outcome of such a process, it is important to study first just one step of the process, namely the interaction of the particle with just one RCS. This was the basic motive behind two recent papers of ours (Efthymiopoulos, Gontikakis and Anastasiadis 2005; Gontikakis, Efthymiopoulos and Anastasiadis 2006) in which we explored the particle dynamics and acceleration in a single Harris-type RCS configuration by numerical and analytical means. A basic outcome of our study is an analytical formula yielding the maximum possible kinetic energy gain of the particles passing through the RCS as a function of the particles' initial kinetic energy and of the physical parameters of the current sheet. Our formula generalizes previous formulae given by Speiser (1965) and Litvinenko (1996), containing the later as asymptotic limits. In the present paper we further generalize this formula to an approximate formula valid for single $\mathrm{X}$-point topologies. Following the assessment of this 'one-step' process, we then pass to modelling the acceleration of particles inside a complex flaring

active region as a stochastic process. In this we repeat the methodology used in Gontikakis, Anastasiadis and Efthymiopoulos (2007) for Harris-type RCSs, and we provide a further theoretical analysis of the results obtained there in.

In summary, the simulations are done as follows: particles are initially interacting with a single RCS. This changes their kinetic energy distribution. After this interaction, the particles are considered to perform a free flight following the magnetic field lines until they reach another RCS present in the active region. This process is in principle repeated ad infinitum. Nevertheless, one finds that the kinetic energy distribution tends to acquire a limiting form following a few such steps, after which no appreciable change is seen in the distribution. This fact was observed in our previous study and we here provide a theoretical explanation for it. Furthermore, we expand our study by considering also multiple encounters of the particles with local X-point reconnecting magnetic field configurations. In this we find again the tendency of the kinetic energy distribution to reach a limiting form. We thus conclude that this phenomenon is probably generic, i.e., independent of the details of the reconnection topology. 
In the next section (Section 2) we outline the basic characteristics of our numerical set up. In Section 3 the interaction of particles with a single RCS is studied, followed by our results for the multiple encounters (Section 4). Section 5 summarizes our results.

\section{The numerical set-up}

We assume two types of magnetic field reconnecting topologies. The first type is the Harris-type model of Litvinenko and Somov (1993) that represents one local current sheet within an active region. The magnetic and electric fields inside one sheet of half-thickness $a$ are given in Equation (1):

$$
\begin{aligned}
& E=(0,0, E) \\
& B=\left(-\mathrm{y} / a, \xi_{\perp}, \xi_{\|}\right) B_{0} \quad \text { for }|\mathrm{y}| \leq a .
\end{aligned}
$$

The edges of the current sheet are at $\mathrm{y}= \pm a$. The magnetic field is normalized in units of $B_{0}$, the value of the main reconnecting component at the edges. The magnetic field has two components, parallel and perpendicular to the current sheet plane, called the 'longitudinal' $\left(\xi_{\|}\right)$and 'transverse' $\left(\xi_{\perp}\right)$ component respectively. The second type is an $\mathrm{X}$-point topology defined by the following equations:

$$
\begin{aligned}
& E=(0,0, E) \\
& B=\left(-\mathrm{y} / a,-(\mathrm{x} / a) \xi_{\perp}, \xi_{\|}\right) B_{0} \quad \text { for }|\mathrm{y}| \leq a
\end{aligned}
$$

Note that a Harris-type configuration is a simplified model for, say, the left domain of an X-point configuration far from the $\mathrm{x}=\mathrm{y}=0$ point.

In Efthymiopoulos, Gontikakis and Anastasiadis (2005), we studied the dynamics of the particles in a Harris-type RCS using the Hamiltonian equations of motions. We found that, using the problem's symmetry, the initial 3-degrees of freedom Hamiltonian can be reduced to a two degrees of freedom Hamiltonian of a form:

$$
H=\frac{1}{2} p_{y}^{2}+\frac{1}{2}\left(c_{4}+\frac{1}{2} y^{2}\right)^{2}+\frac{1}{2}\left(I_{2}-\xi_{\perp} z+\xi_{\|} y\right)^{2}-\epsilon z
$$

with $\epsilon=E m /\left(a B_{0}^{2} e\right)$ being the normalized electric field ( $m$ and $e$ are the particle's mass and charge) and $x=\mathrm{x} / a, y=\mathrm{y} / a$ are the normalized space coordinates. The time coordinate, implicit in Equation (3), is scaled with the gyration period. The canonical momenta are mapped to velocities via $p_{y}=\dot{y}, c_{4}=\dot{z}-\frac{1}{2} y^{2} . I_{2}$ is an integral of motion yielding the velocity of the $\mathrm{x}$-component of motion (missing in Equation (3)), namely:

$$
I_{2}=\dot{x}-\xi_{\|} y+\xi_{\perp} z
$$

In addition, we found (Efthymiopoulos, Gontikakis and Anastasiadis 2005; Gontikakis, Efthymiopoulos and Anastasiadis 2006) that the maximum kinetic energy gain for one particle can be expressed as a function of the field parameters, the position of injection into the sheet and the initial energy of the particle with the relation:

$$
E_{\max }=\frac{\epsilon}{\xi_{\perp}^{2}}\left(\xi_{\perp} I_{2}+\xi_{\|} \xi_{\perp} y_{\text {out }}+\epsilon+\sqrt{2 \xi_{\perp} I_{2} \epsilon+2 \xi_{\|} \xi_{\perp} y_{\text {out }} \epsilon+\epsilon^{2}+2 \xi_{\perp}^{2} E_{0}}\right)
$$


where $E_{0}$ is the initial kinetic energy of a particle injected at $y=y_{0}$, the value of $I_{2}$ is set equal to $I_{2}=\dot{x}_{0}-\xi_{\|} y_{0}$, and the exit of the particle is through the edge $y=y_{\text {out }}$.

The study of the particle orbits in a Harris-type current sheet given by Equation ( 1 ) is performed by integrating numerically the relevant equations of motions using the Hamiltonian of the form given in Equation (3). For the case of an X-point geometry we integrate the Newtonian equations of motion using Equation (2) for the electromagnetic field. In all our numerical simulations we consider that the RCS half-thickness $a$ is the unit of length and the inverse gyrofrequency $\omega_{B}^{-1}=m / q B_{0}$ is the unit of time. For a typical value of the main magnetic field of $B_{0}=100 G$, $\omega_{B}^{-1} \simeq 6 \times 10^{-10} \mathrm{sec}$ for electrons and $10^{-6} \mathrm{sec}$ for protons. A super-Dreicer electric field $E$ of $100 \mathrm{~V} / \mathrm{m}$ is used, which corresponds to a normalized field of $\epsilon=10^{-5}$ for electrons and of $\epsilon=\left(m_{p} / m_{e}\right) \times 10^{-5} \simeq 1.84 \times 10^{-2}$ for protons. The numerical integration is carried out, for both geometries, until the particle reaches the edges of the reconnection site which is at at $y= \pm 1$. Finally we consider particle injections at three different positions along the y-axis, i.e., from the edges $(y= \pm 0.9)$ or from the central plane $(y=0)$.

\section{Particle interactions by single RCS}

Particles can follow several types of orbits inside reconnecting magnetic fields. The form of the orbit depends strongly on the value of the longitudinal component of the magnetic field $\xi_{\|}$. When $\xi_{\|}$is less than about 0.1, electrons can follow chaotic orbits which lead them to escape, or regular quasi-periodic orbits along which the particle remains trapped in the sheet. In Figure 1 two kinds of electron orbits are shown inside a RCS. In the first case (panels a,b) the particle never reaches the $y= \pm 1$ edges and performs a quasi-periodic motion, mirrored by the growth of the magnetic field strength away from the $y=0$ plane. The second orbit (panels c,d) is an escaping chaotic orbit. Initially the electron oscillates in the y-axis with $|y|<0.05$. Later however, it leaves the current sheet by moving along a magnetic field line. For large values of $\xi_{\|}$most particles follow adiabatic orbits. In that case the particles nearly follow adjacent magnetic field lines before escaping from the accelerating site.

Chaotic and regular (adiabatic) orbits are also found in the case of acceleration through an X-point configuration. In Figure 2 an electron moves chaotically through an X-point with $\xi_{\|}=0, \xi_{\perp}=10^{-3}$ and $\epsilon=10^{-5}$. The electron starts its motion at $\left(x_{0}, y_{0}\right)=(0,0.9)$ and, then drifts toward the $\mathrm{X}$-point line at $(x, y)=(0,0)$. There, it performs a bounce motion which pushes it to larger values of $y$ until it reaches the $y=1$ edge where it escapes from the current sheet.

We now consider the acceleration of $\mathrm{N}=30000$ particles (electrons or protons) that form initially a thermal distribution at a typical coronal temperature of $2 \times$ $10^{6}$ Kelvin. This means that the velocities of particles are randomly oriented and form a Maxwellian distribution. Each particle enters from one of three selected injection points along the $\mathrm{y}$-axis, namely $y= \pm 0.9$ or $y=0$. The initial position of particles on the $\mathrm{x}$-axis is defined as $|x|<0.5$ for X-points and $x=0$ for Harristype current sheets. The field parameters, for both current sheets configurations, are $\xi_{\|}=1, \xi_{\perp}=10^{-3}$ with dimensionless electric field parameters $\epsilon=10^{-5}$ for electrons and $\epsilon=1.84 \times 10^{-2}$ for protons. 

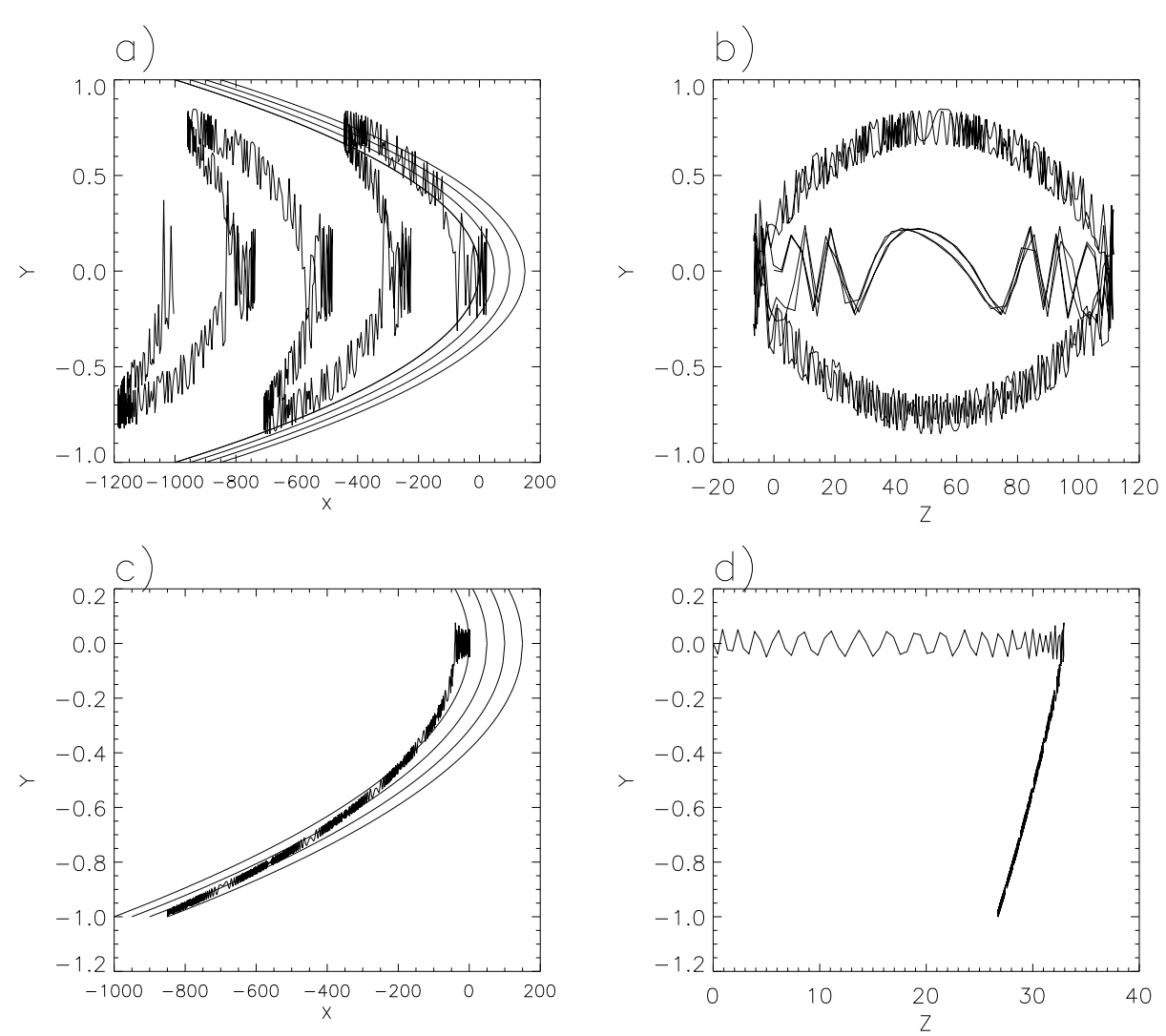

Figure 1. Examples of single electron orbits inside a Harris - type reconnecting current sheet with $\xi_{\|}=0$ and $\xi_{\perp}=10^{-3}$. In panels (a) and (b) the projections of a trapped (mirrored) trajectory are shown. In panels (c) and (d) the projections of an escape orbit are presented. The continues lines in panels (a) and (c) are visualizations of the magnetic field lines.

In Figure 3, we present a comparison between particles accelerated through a Harris-type current sheet and particles accelerated through an X-point. The presented characteristics are the final kinetic energy distribution and the final pitch angle distribution of the particles. In all the kinetic energy distribution plots, the dotted vertical lines correspond to the maximum kinetic energy gain computed through Equation (5). Electrons kinetic energy distributions have a short energy range that is characterized by a number of energy peaks. In the Harris- type case three energy peaks correspond to the initial injection positions. In the case of X-points, the distribution presents two peaks, one for particles injected from the sides $(y= \pm 0.9)$ and one, with higher energy, for injection from the X-point center. Moreover, for the same parameter values used in both geometries, particles accelerated with an X-point gain less kinetic energy by a factor of $\simeq 10$. Because of the fact that the particles entry points are very localized in the $y$-axis, the resulting final kinetic energy distributions are peaked in a short range of final energies rather than yielding a power-law (like in Wood and Neukirch (2005); our case best compares with Figure 5 of that paper, which corresponds to particles accelerated near the separatrix). On the other hand, our injection of particles at $y=0$ is representative of particles being accelerated 


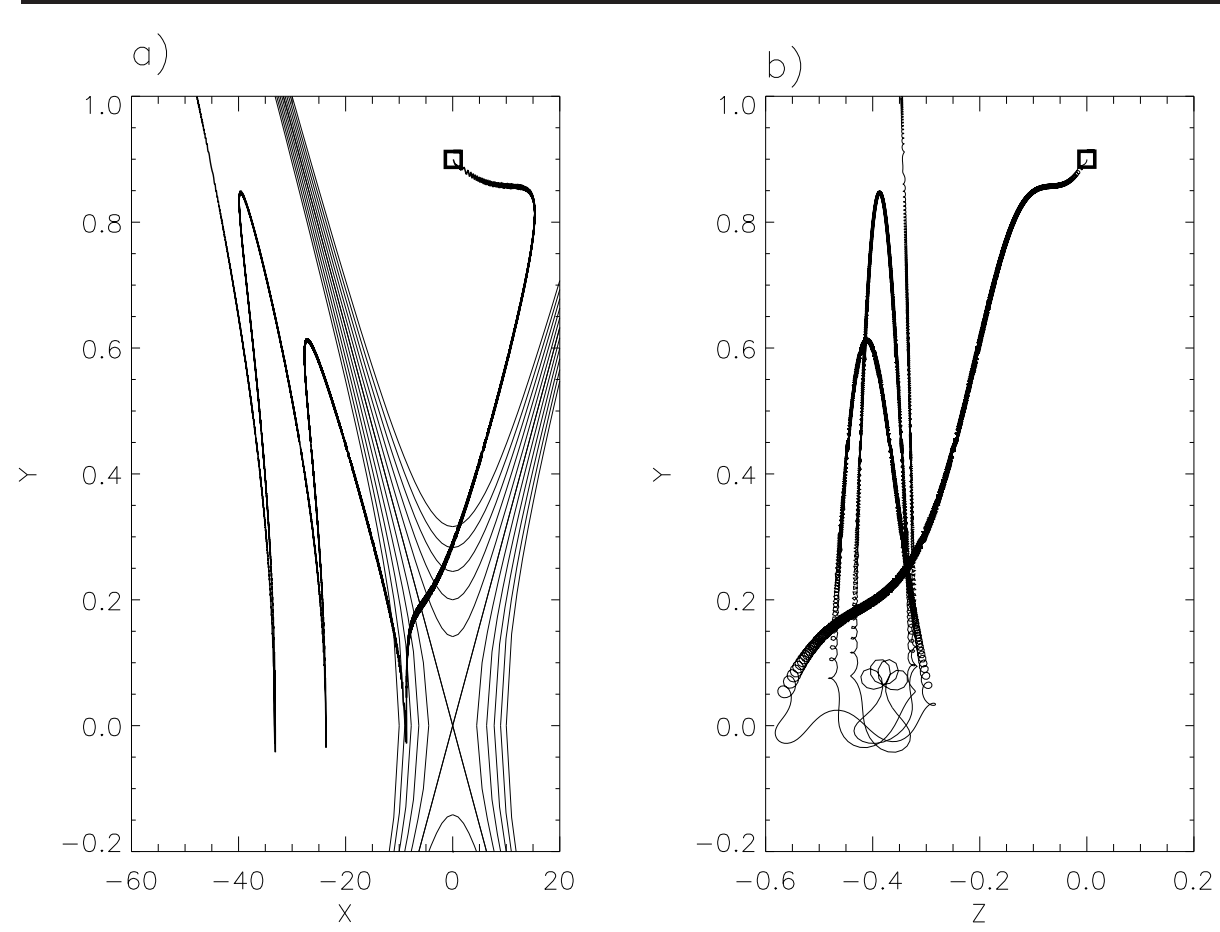

Figure 2. Example of single electron orbit inside an X-point configuration, with $\xi_{\|}=0$ and $\xi_{\perp}=10^{-3}$. The continuous lines in panel (a) are visualizations of the magnetic field lines.

when they start already inside the current sheet. In fact, if a uniform distribution of initial conditions is taken in the range $-0.9 \leq y \leq 0.9$ the final kinetic energy distribution fills the gaps between the peaks of Figure 3 a,c.

The analytic formula of Equation (5) predicts well the numerical result, especially in the case of the Harris-type (Gontikakis, Anastasiadis and Efthymiopoulos 2007). In the case of the acceleration through the X-point on the other hand, the analytical solution gives a reasonable estimation for the particles entering from the above edge if we replace the parameter $\xi_{\perp}$ with an effective value (see below). As the particle moves along the $\mathrm{x}$-axis it encounters an increasing value of the perpendicular component of the magnetic field $B_{y}=\xi_{\perp} x$. Roughly one can replace $\xi_{\perp}$ with $\xi_{\perp}\langle x\rangle(\langle x\rangle$ is the average position along the $\mathrm{x}$-axis of a particle orbit) in Equation (5) which gives the right estimation of the kinetic energy gain. The analytic expression is consistent with the numerical results as long as the particles enter from the sides of the X-point and is slightly short in energy for electrons starting at the X-point center (panel c). In fact, as already mentioned, in the adiabatic limit the projection of the particles' orbits on the $x-y$ plane follows essentially the projection of the magnetic field lines on the same plane. The latter is given by the family of hyperbola:

$$
y^{2}-\xi_{\perp} x^{2}=\text { const }
$$

Particles entering the sheet at $\left(x_{0}, y_{0}\right)$ and leaving the sheet at $x_{\text {out }}, y_{\text {out }}$ then satisfy approximately the relation $\left|x_{\text {out }}\right| \simeq\left(1 / \sqrt{\xi_{\perp}}\right) \sqrt{y_{\text {out }}^{2}-y_{0}^{2}-\xi_{\perp} x_{0}^{2}}$. The average 
Harris type
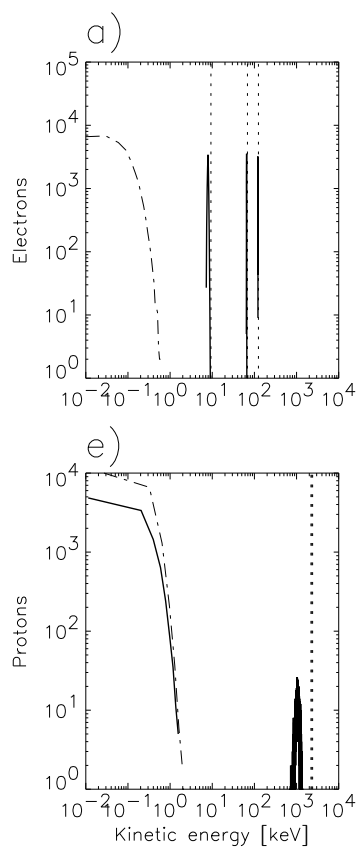

b)
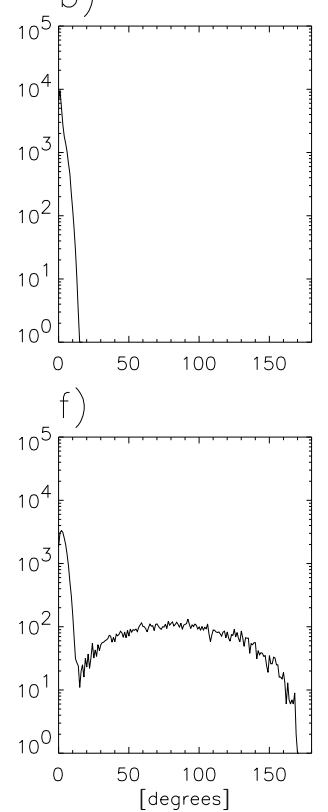

$x$-point
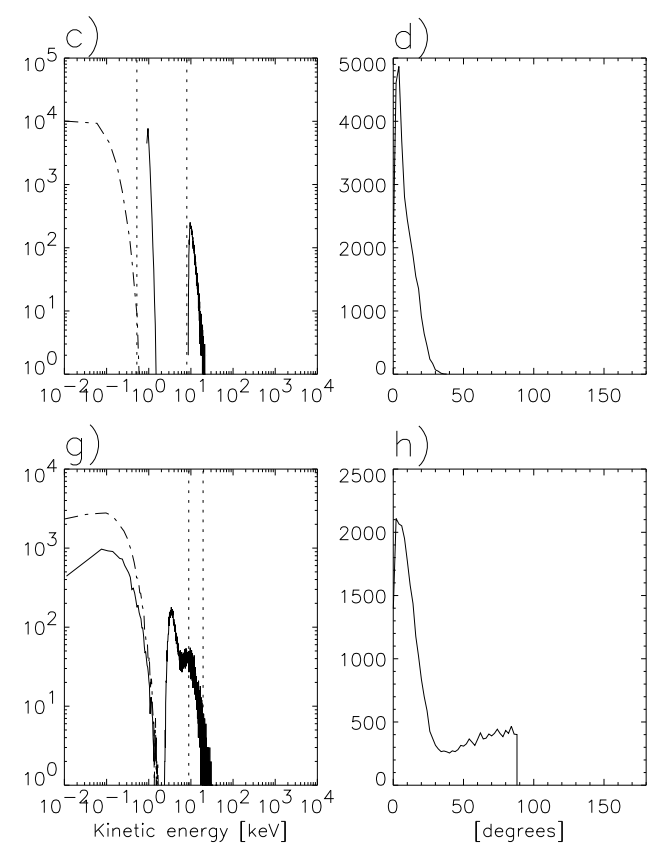

Figure 3. Distributions of kinetic energy and pitch angles for a Harris - type (panels a,b, e and f) and X-point (panels c, d, g and h) RCS with $\xi_{\|}=1$ and $\xi_{\perp}=10^{-3}$. The first row is for electrons and the second row is for protons. In panels (a), (c), (e) and (g) the dashed line corresponds to the initially injected kinetic energy distribution and the vertical dashed lines to maximum kinetic energy derived by the analytical formula given by Equation (5)

transverse magnetic field 'felt' by the particles can then be estimated as:

$$
<\xi_{\perp}>\approx \frac{\xi_{\perp}}{2}\left(\left(1 / \sqrt{\xi_{\perp}}\right) \sqrt{y_{\text {out }}^{2}-y_{0}^{2}-\xi_{\perp} x_{0}^{2}}-x_{0}\right) .
$$

Equation (5) can then be used to estimate analytically the maximum amount of kinetic energy gain for the particles crossing an X-point, if $<\xi_{\perp}>$ of Equation (7) is substituted in the place of $\xi_{\perp}$ in (5).

Protons present a behavior which is quite common in both the Harris-type and $\mathrm{X}$-point types of accelerators. In particular, a large fraction of the initially injected protons are not accelerated, but they simply cross the current sheet practically without changing their kinetic energy. The final kinetic energy distribution of these protons is thus still a Maxwellian (see Figure 3 panels e and g). On the other hand, likewise electrons, the kinetic energy distributions of accelerated protons present secondary features due also to the three different positions of injection. Nevertheless, such features are less apparent because the overall distribution is broader for protons than for electrons. In the case of X-point, the kinetic energy distribution of the accelerated protons presents two components, one associated with an injection from the sides and the other one for injection from the center of the current sheet.

In a Harris-type, the final kinetic energy of protons is of order $1 \mathrm{MeV}$, which is 10 times larger than the energy gain of electrons. In the case of an X-point, protons and 
a)

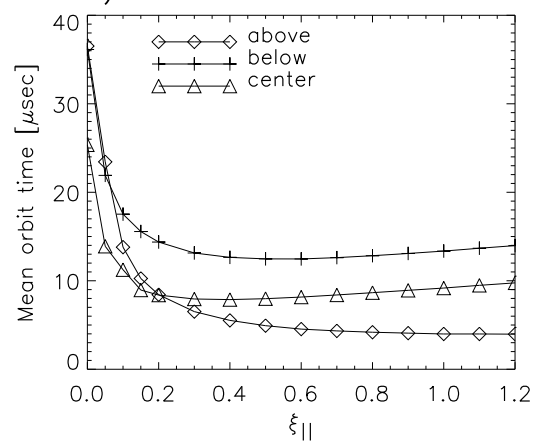

c)

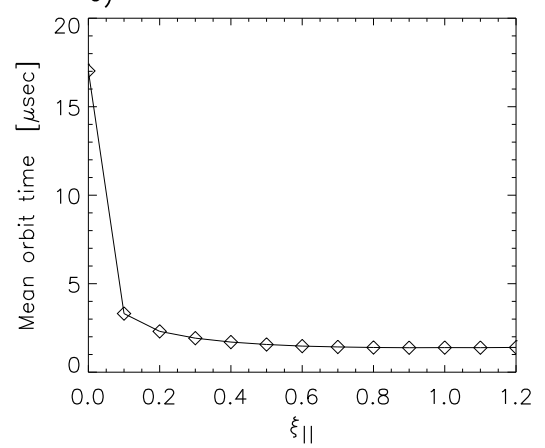

b)

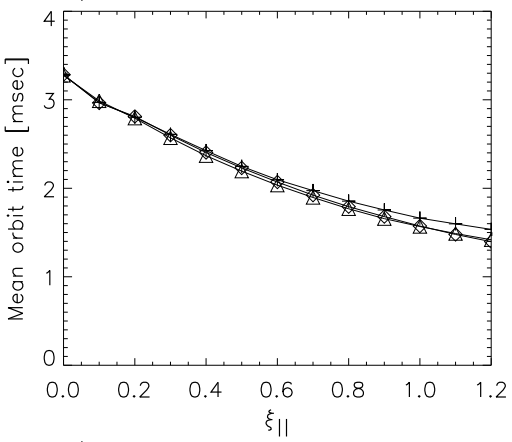

d)

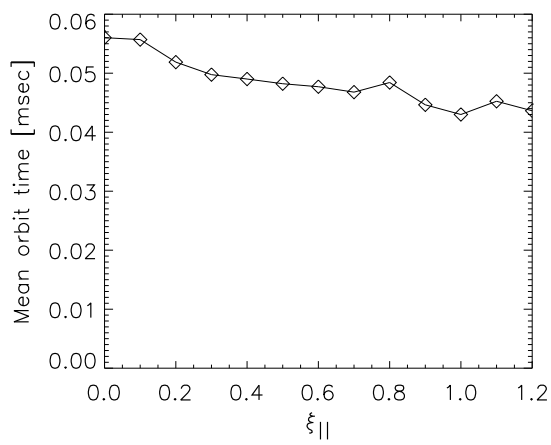

Figure 4. Time spend by accelerated particles inside $(|y|<1)$ a current sheet depending on the value of the $\xi_{\|}$with $\xi_{\perp}=10^{-3}$. The first row concerns acceleration through a Harris-type and the second row, acceleration through a X-point. In panels (a) and (c) the particles are electrons and in panels (b) and (d) protons. At the first row, diamonds shows injections from above, crosses injections from below and triangles from the center of the current sheet. Particles are injected from the sides of X-points. The electric field is of $10^{-5}$ and $1.84 \times 10^{-2}$ for electrons and protons respectively.

electrons are both seen to gain considerably less energy, of order $10 \mathrm{keV}$. As regards exit pitch angle distributions, both electrons and protons, accelerated in either Xpoints or Harris-types current sheets, present similar narrow distributions (Figure 3 $\mathrm{b}, \mathrm{d}, \mathrm{f}, \mathrm{h})$. The pitch angle distributions of protons exhibit a broad band corresponding to the population of non accelerated particles, the velocities of which are randomly oriented both before and after the interaction with the RCS.

Finally, we studied the time needed for a particle to leave a current sheet as a function of the longitudinal magnetic field component $\xi_{\|}$. The time needed for an electron to leave the current sheet is of the order or a few micro seconds. For protons the time is of some milliseconds (Gontikakis, Anastasiadis and Efthymiopoulos 2007). Note that such time scales are still out of reach of the time resolution of present instruments thus little can be said as regards comparison of such figures with observations. In order to compute the mean time needed for the particles to reach the edges of current sheets (at $y= \pm 1$ ), we followed the orbits of 1000 protons and electrons through a Harris-type and an X-point current sheet for values of the $\xi_{\|}$in the range (0 to 1.2). In Figure 4 we present the mean time as a function of the value of $\xi_{\|}$for electrons (panel a) and protons (panel b). For protons, the time inside 
the current sheet is a decreasing function of $\xi_{\|}$. For electrons, the acceleration time decreases sharply when $\xi_{\|}$changes from 0 to 0.1 . This function also depends on the injection point. Electrons entering the current sheet from above have acceleration times slowly decreasing with $\xi_{\|}$. On the other hand, electrons initiated from the center or entering from below $(y=-0.9)$ show a small rise of the acceleration time as a function of $\xi_{\|}$. In the case of electrons, when $y_{0} \neq 0.9$ the acceleration time grows asymptotically, for large $\xi_{\|}$, as a function of $\xi_{\|}$. This result is in agreement with the asymptotic analysis of Litvinenko (1996)in the case of strongly magnetized particles.

\section{Particle interactions by multiple RCS}

As already stated in the introduction, an appropriate description of the environment within which the particle acceleration takes place involves considering the coexistence of multiple reconnecting current sheets. In this section, we present our numerical results for the interaction of electrons and protons with multiple current sheets taking parameter values stochastically from a homogeneous distribution, together with a theoretical analysis of these results.

\subsection{Numerical results}

We performed numerical simulations of particles interacting subsequently with 15 current sheets. In a previous work (Gontikakis, Anastasiadis and Efthymiopoulos 2007) we studied the cases of electrons and protons accelerated successively from Harris-type current sheets. In the present work we present the acceleration of protons and electrons from X-point reconnecting current sheets. We consider 5000 particles that have initially a thermal kinetic energy at $2 \times 10^{6} \mathrm{~K}$. They are injected inside the first X-point of the series. The injection points of each particle is at $y= \pm 0.9$ and their initial position along the $\mathrm{x}$-axis varies randomly inside the range $-0.5<x<$ 0.5. The particles with modified kinetic energies after the interaction with the first $\mathrm{X}$-point are injected through the next RCS. This procedure is repeated 15 times. The parameters $\xi_{\|}$and $\xi_{\perp}$ for each RCS are selected randomly in the range: $10^{-4}<\xi_{\perp}<$

$10^{-2}, 0.01<\xi_{\|}<1$. Similarly the normalized electric field is randomly selected in the range $10^{-6}<\epsilon<5 \times 10^{-5}$ for electrons and $1.84 \times 10^{-3}<\epsilon<9.2 \times 10^{-2}$ for protons. The orientation of the accelerated particles velocities is randomized every time before their injection into the next current sheet.

The resulting distributions are presented in Figure 5 for electrons and in Figure 6 for protons. The main result found again is that the evolution of the kinetic energy distribution converges towards a final state after a small number of current sheets encounters. This happens for both types of particles and in both types of current sheet configurations. In Figures. 5, 6 in panel (p) one can compare the last three kinetic energy distributions (13th, 14th and 15th) and conclude that have the same shape at high energies. The same result was also found for the case of multiple encounters with Harris-type current sheets (see Gontikakis, Anastasiadis and Efthymiopoulos 2007).

For the case of X-points, the maximum kinetic energy gain is of the order of $10 \mathrm{keV}$ for electrons and of $100 \mathrm{keV}$ for protons. In Figure 7 one can compare the final kinetic energy distributions for multiple encounters with Harris-type (panels 

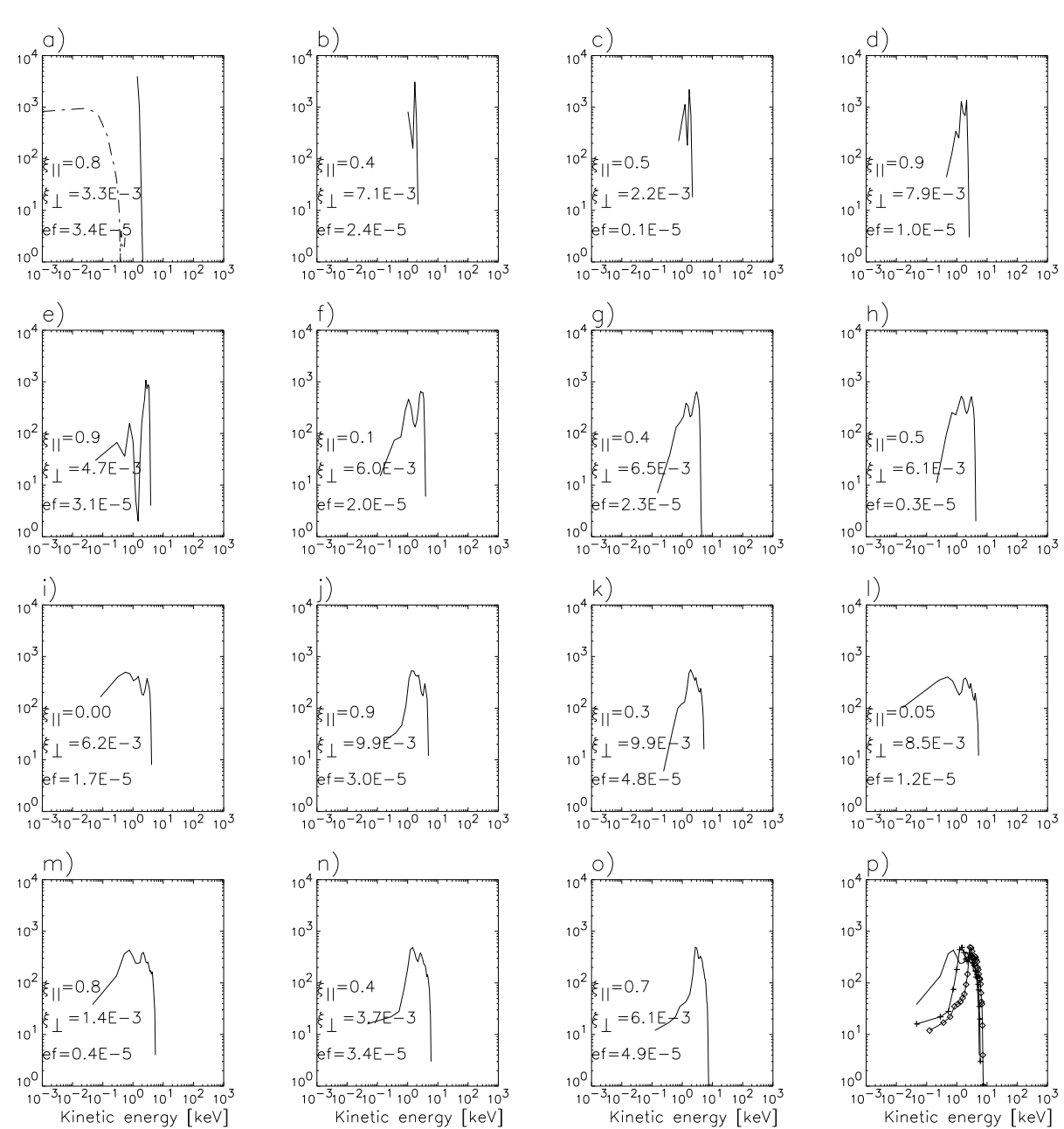

Figure 5. Consecutive acceleration of 5000 electrons, initially with thermal velocities from 15 $\mathrm{X}$-points. Each panel from left to right and from top to bottom shows the final kinetic energy distribution of the electrons after the interaction with each X-point. In the first panel, the dashed curve is the initial thermal kinetic energy distribution. Panel (p) shows the three last distributions on the same panel.

a,e) with the X-points (panels c,g). As was the case for single encounters, particles through Harris-type reconnecting current sheets, gain more energy by a factor of 10 in respect to energy gain through their interactions with X-point current sheet configuration.

\subsection{Theoretical analysis}

In Gontikakis, Anastasiadis and Efthymiopoulos (2007) a heuristic explanation was given for the asymptotic convergence of the kinetic energy distribution in a multiple particle- RCS interaction. The explanation was that as the input kinetic energy increases, the probability of an RCS, with random parameters $\left(\xi_{\perp}, \xi_{\|}, \epsilon\right)$ within a 

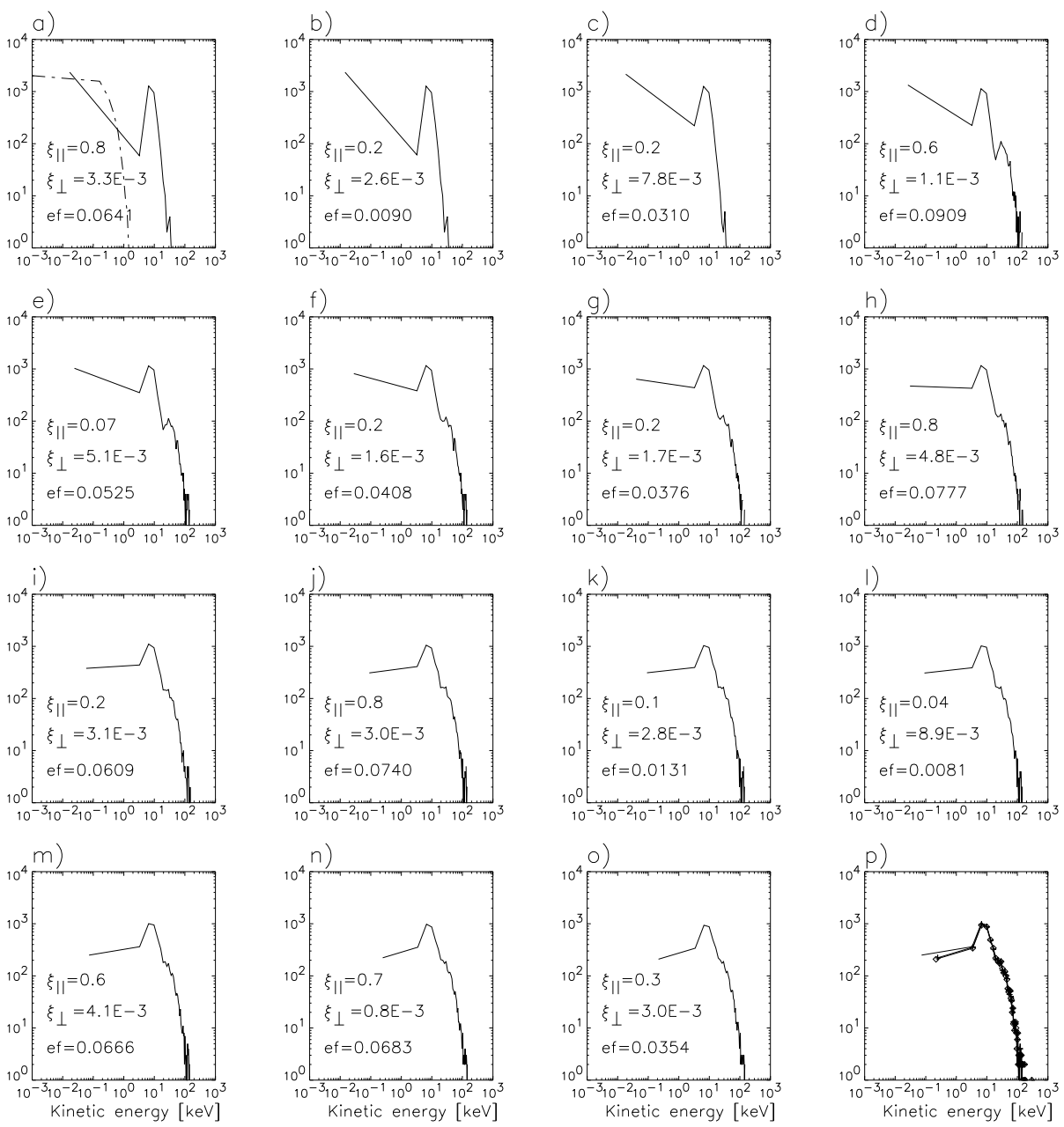

Figure 6. Same as for Figure 5 for protons.

specified parameter space, to be an efficient accelerator decreases. We hereby provide a detailed theoretical treatment of these results.

Let $w_{0}$ be the maximum percentage of kinetic energy gain for particles with initial energy $E_{0}$ interacting with a Harris-type RCS with parameters $\left(\xi_{\perp}, \xi_{\|}, \epsilon\right)$. We shall consider in detail the case of particles entering the RCS at $z=0$ with $\dot{x}_{0}=0$. Thus $I_{2}=-\xi_{\|} y_{0}$. Setting $E_{\max }=w_{0} E_{0}$ in Equation (5) yields:

$$
\xi_{\perp} \xi_{\|} \Delta y+\epsilon+\sqrt{2 \epsilon \xi_{\perp} \xi_{\|} \Delta y+\epsilon^{2}+2 \xi_{\perp}^{2} E_{0}}=\frac{w_{0} \xi_{\perp}^{2} E_{0}}{2}
$$

where $\Delta y=y_{\text {out }}-y_{0}$. Equation (8) can be used in order to find the critical value of $\xi_{\|}$above which the kinetic energy gain surpasses the percentage $w_{0}$ for fixed values of the remaining parameters. Setting $\xi_{\|}=0$ in Equation (8) yields the minimum 


\section{Harris type}

a)
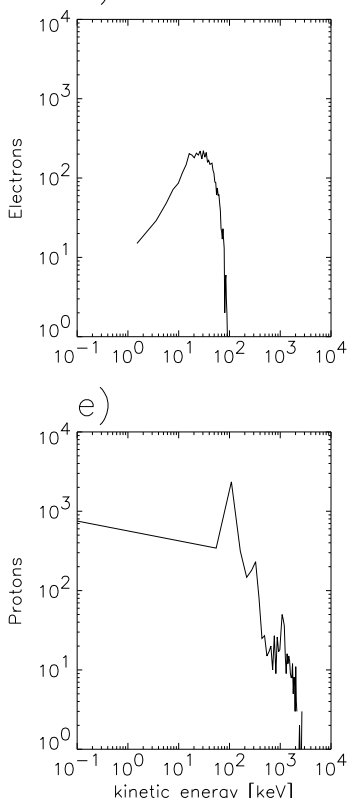

b)
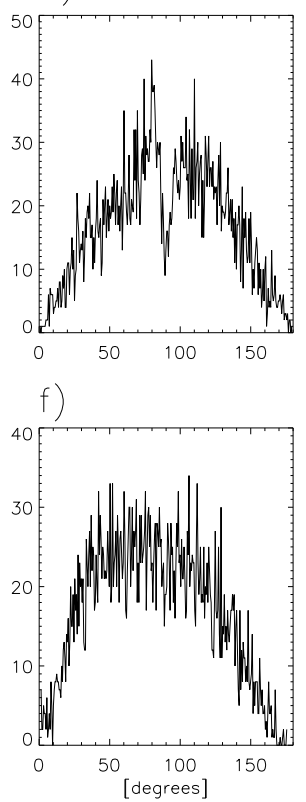

$x$-points
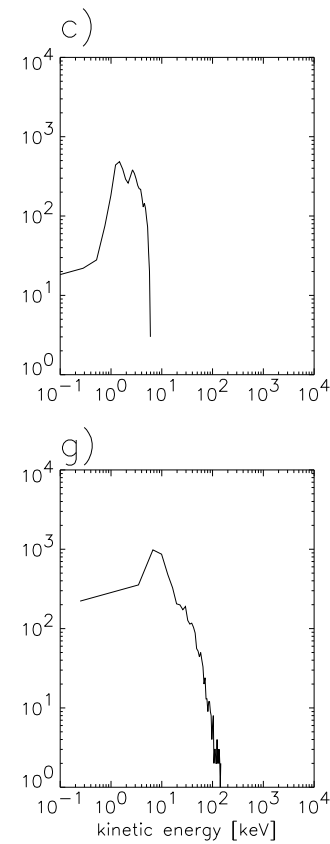

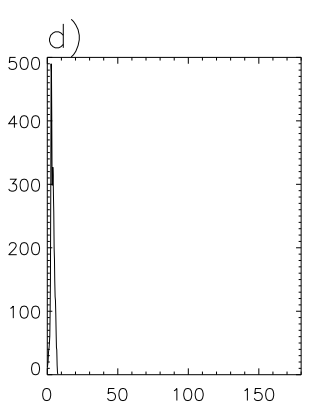

h)

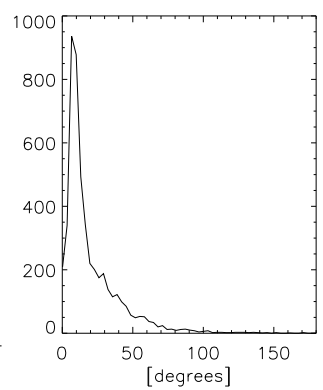

Figure 7. Distributions of kinetic energy and pitch angles of multiple encounters of protons and electrons with Harris-type (panels a,b, e and f) and X-point (panels c, d, g and h) current sheets. The first row is for electrons and the second row is for protons. Panels a,c,e,g shows the final kinetic energy of the particles whereas panels $\mathrm{b}, \mathrm{d}, \mathrm{f}, \mathrm{h}$ the final pitch angle distributions.

percentage:

$$
w_{m}=\frac{\epsilon}{\xi_{\perp}^{2} E_{0}}\left(\epsilon+\sqrt{\epsilon^{2}+2 \xi_{\perp}^{2} E_{0}}\right) .
$$

If $w_{0}>w_{m}$ Equation (8) admits a positive solution for $\xi_{\|}$provided that:

$$
\xi_{\|} \leq \frac{w \xi_{\perp} E_{0}}{\epsilon \Delta y}-\frac{\epsilon}{\xi_{\perp} \Delta y}
$$

The solution reads:

$$
\xi_{\|}=\frac{1}{\Delta y}\left[\frac{w_{0} \xi_{\perp} E_{0}}{\epsilon}-\sqrt{2\left(w_{0}+1\right) E_{0}}\right] .
$$

If $\xi_{\|}$is greater than the value (11), the percentage of kinetic energy gain is larger than $w_{0}$. We now wish to estimate, for fixed $\left(w_{0}, E_{0}\right)$ the probability within the parameter space that an RCS will accelerate the particles by a percentage larger or equal than $w_{0}$. This can be done with the help of Figure 8. The square box shows the domain of the parameter space for $\epsilon$ and $\xi_{\perp}$, which in our simulations is $10^{-6} \leq \epsilon \leq 3 \times 10^{-5}$, 
$10^{-3} \leq \xi_{\perp} \leq 10^{-2}$. The lower solid line corresponds to the equation:

$$
\xi_{\perp}=\frac{\epsilon}{w_{0}} \sqrt{\frac{2\left(w_{0}+1\right)}{E_{0}}}
$$

This line yields the locus of values of $\left(\epsilon, \xi_{\perp}\right)$ for which the given percentage $w_{0}$ coincides with the minimum possible percentage of kinetic energy gain for the given energy $E_{0}$, according to Equation (9). This locus divides the square into two domains. In the lower domain $\left(D_{1}\right)$ one has $w_{m}>w_{0}$, thus for all values of $\xi_{\|}$the kinetic energy gain is higher than $w_{0}$. On the other hand, in the upper domains $\left(D_{2}\right.$ and $\left.D_{3}\right)$ one has $w_{m}<w_{0}$, thus only for $\xi_{\|}$large enough the percentage $w_{0}$ can be surpassed. Now, the upper solid line yields the locus at which the minimum value of $\xi_{\|}$for which the percentage $w_{0}$ is reached exceeds the upper allowed value $\xi_{\|, \max }$ within the selected parameter space (in our examples $\xi_{\|, \max }=1$ ). This is given by

$$
\xi_{\perp}=\frac{\epsilon}{w_{0}}\left(\sqrt{\frac{2\left(w_{0}+1\right)}{E_{0}}}+\frac{\Delta y}{w_{0} E_{0}}\right)
$$

In the domain $D_{3}$ above the line (13) we have $\xi_{\|}>\xi_{\|, \max }$, thus this domain lies entirely outside the parameter space. In view of the above analysis, the total subvolume of the parameter space in which the kinetic energy gain exceeds the percentage $w_{0}$ is given by

$$
V\left(w_{0}, E_{0}\right)=\left(\xi_{\|, \max }-\xi_{\|, \min }\right) S\left(D_{1}\right)+\int_{D_{2}}\left(\xi_{\|, \max }-\xi_{\|}\left(w_{0}, E_{0}, \xi_{\perp}, \epsilon\right)\right) d \xi_{\perp} d \epsilon
$$

where $\xi_{\|, \max }, \xi_{\|, \min }$ are the limits of $\xi_{\|}$in the selected parameter space, $S\left(D_{1}\right)$ is the surface of the domain $D_{1}$ and $\xi_{\|}\left(w_{0}, E_{0}, \xi_{\perp}, \epsilon\right)$ is the solution (11) for $\xi_{\|}$. The probability of an RCS to accelerate the particles by a gain factor larger than $w_{0}$ is given by:

$$
P\left(w_{0}, E_{0}\right)=\frac{V\left(w_{0}, E_{0}\right)}{V_{\text {total }}} .
$$

This probability is roughly proportional to the total area of the domains $D_{1}$ and $D_{2}$ over the total area of the square box. We can see now that as $E_{0}$ increases, the probability $P\left(w_{0}, E_{0}\right)$ decreases for any fixed value of $w_{0}$. This is simply because, according to Eqs.(12) and (13) the slopes of both limiting lines decrease, thus the total area $S\left(D_{1}\right)+S\left(D_{2}\right)$ also decreases. In fact, beyond a sufficiently high value of $E_{0}$, at which the upper limiting line touches the lower right apex of the square, the domains $D_{1}$ and $D_{2}$ both shrink to a null domain. Thus the probability of a gain factor $w_{0}$ shrinks to zero. This was precisely found numerically in Figure 9 of Gontikakis, Anastasiadis and Efthymiopoulos (2007) (note an error in this figure: $w$ there corresponds to $w_{0}-1$ in our notation). Setting the value $w_{0}=1$ (corresponding to $w=0$ in Gontikakis, Anastasiadis and Efthymiopoulos 2007), as well as $\xi_{\perp}=$ $10^{-3}$ and $\epsilon=3 \times 10^{-5}$ for the lower right appex of the square, and $\xi_{\|, \max }=1$, we find a maximum energy through Equation (8) equal to $E_{0} \simeq 7.5 \times 10^{-2}$, or, in physical units $E_{0}=4.2 \times 10^{2} \mathrm{keV}$, which agrees well with the limit found numerically in Gontikakis, Anastasiadis and Efthymiopoulos (2007). 


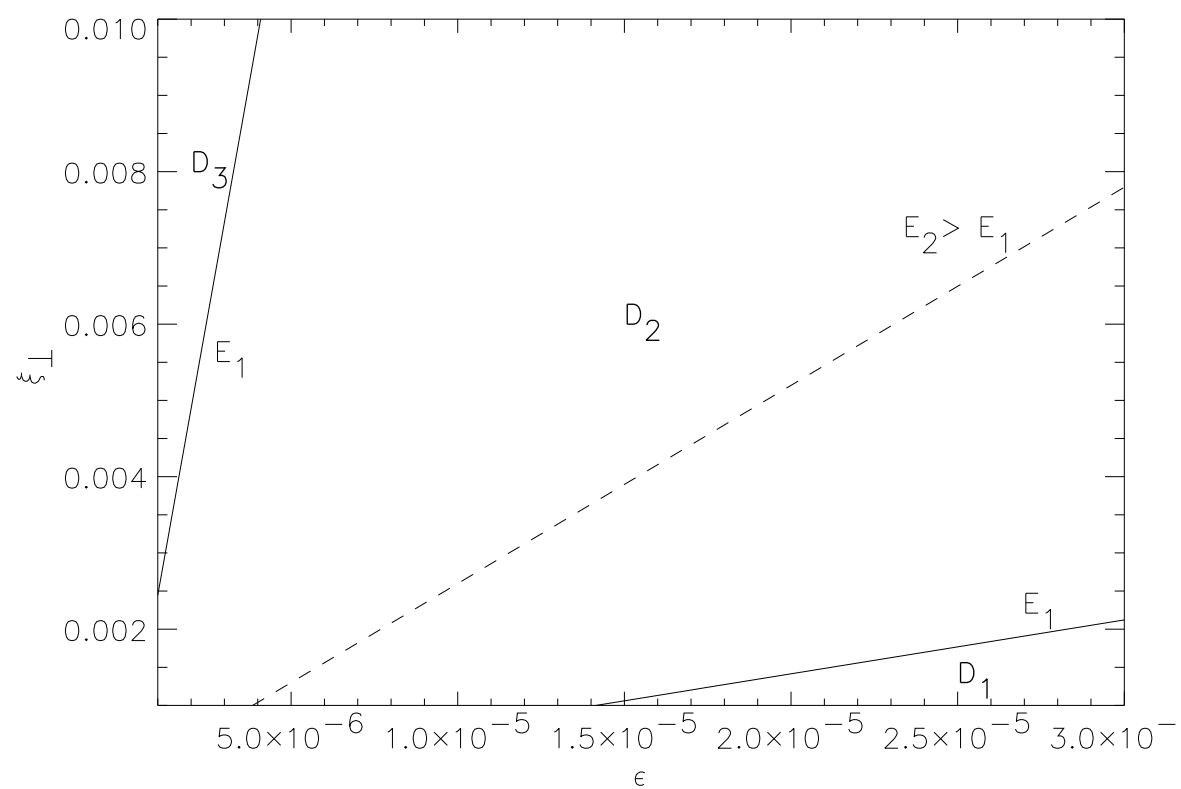

Figure 8. The square box $10^{-6} \leq \epsilon \leq 3 \times 10^{-5}, 10^{-3} \leq \xi_{\perp} \leq 10^{-2}$. The lower and upper solid lines are the graphs of Eqs. (12) and (13) respectively for $w_{0}=1, E_{0}=E_{1}=4.7 \mathrm{keV}$. A kinetic energy gain $w_{0} E_{1}$ is possible for all values of $\xi_{\|}$in the domain $D_{1}$ and for some values of $\xi_{\|}$in the domain $D_{2}$, while it is not possible in the domain $D_{3}$. The dashed line is the graph of Equation (13) for $w_{0}=1$ and $E_{0}=E_{2}=47 \mathrm{keV}$.

\section{Conclusions}

In the present work we studied the acceleration of electrons and protons in a multiple particle-RCS interaction scenario, assuming two different reconnection topologies, the Harris-type current sheet and the X-point. Our main conclusions are the following:

1) The particles accelerated close to a X-point, with specific field parameters $\left(\xi_{\perp}, \xi_{\|}, \epsilon\right)$ gain a smaller amount of kinetic energy than particles accelerated through a Harris-type current sheet with the same model parameters. This is because particles accelerated through an X-point encounter a stronger average perpendicular magnetic field component.

2) The particles escape from a Harris-type current sheet in longer times than from an X-point.

3) A previously derived analytical formula (Equation 5), yielding the maximum kinetic energy gain for particles accelerated through a Harris type topology (Gontikakis, Efthymiopoulos and Anastasiadis 2006), provides a satisfactory approximation to the kinetic energy gains computed numerically. (Equation 5) can also be modified to yield an estimate for the kinetic energy gain of particles accelerated by an X-point if the perpendicular component $\xi_{\perp}$ is replaced by an effective field $\left\langle\xi_{\perp}\right\rangle$ which is the mean perpendicular magnetic field along an orbit. 
4) A number of similarities are also found for the acceleration of particles through Harris-type RCSs and X-points. An important fraction of protons with initially thermal distributions are not accelerated at all. The pitch angle distributions of the accelerated electrons and protons, present sharp peaks for angles $<10^{\circ}$.

5) When the particles encounter multiple RCSs with parameters obtain randomly from a uniform sample, the particles kinetic energy distribution tends to acquire a limiting form after some iterations. This tendency was previously observed (Gontikakis, Anastasiadis and Efthymiopoulos 2007) in the case of multiple encounters with Harris-type RCSs, and we here confirm it also in the case of X-points. A theoretical explanation is given by considering the probability of an RCS to act as efficient accelerator (parameterized by the percentage $w_{0}$ of kinetic energy gain with respect to some initial energy $E_{0}$ ). In particular we demonstrate analytically that this probability decreases as $E_{0}$ increases.

Acknowledgements We wish to thank Dr. I. Contopoulos for stimulating discussions on the problem of magnetic reconnection as well as the anonymous referee and Dr. K-L. Klein for their comments and suggestions that improved our manuscript. The work of CG and CE was supported in part by the Research Committee of the Academy of Athens. AA would like to thank the members of the Particle Acceleration Working Group of the CESRA-2007 workshop for many stimulating discussions.

\section{References}

Anastasiadis, A.: 2002, J. Atmos. Sol. Terr. Phys., 64, 481.

Anastasiadis, A., Vlahos, L.: 1991, Astron. Astrophys., 245, 271.

Anastasiadis, A., Vlahos, L.: 1994, Astrophys. J., 428, 819.

Anastasiadis, A., Vlahos, L., Georgoulis, M.K.: 1997, Astrophys. J., 489, 367.

Anastasiadis, A., Gontikakis, C., Vlimer, N., Vlahos, L.: 2004, Astron. Astrophys., 422, 323.

Aschwanden, M.J., Montello, M., Dennis, B.R., Benz, A.O.: 1995, Astrophys. J., 440, 394.

Aschwanden, M.J., Tarbell, T.D., Nightingale, R.W., Schrijver, C.J., Title, A., Kankelborg, C.C., et al.: 2000, Astrophys. J., 535, 1047.

Benz, A.O.: 1985, Solar Phys., 96, 357.

Benz, A.O., Kosugi, T., Aschwanden, M.J., Benka, S.G., Chupp, E.L., Enome, S., et al.: 1994, Solar Phys., 153, 33.

Browning, P.K., Vekstein, G.E.: 2001, J. Geophys. Res., 106, 18677.

Bruhwiler, D.L., Zweibel, E.G.: 1992, J. Geophys. Res., 97, 10825.

Bulanov, S.V.: 1980, Sov. Astron. Lett., 6, 206.

Burkhart, G.R., Drake, J.F., Chen, J.: 1990, J. Geophys. Res., 95, 18833.

Craig, I.J.D., Litvinenko, Y.E.: 2002, Astrophys. J., 570, 387.

Crosby, N.B., Aschwanden, M.J., Dennis, R.B.: 1993, Solar Phys., 143, 275.

Crosby, N.B., Vilmer, N., Lund, N., Sunyaev, R.: 1998, Astron. Astrophys., 334, 299.

Dalla, S., Browning, P.K.: 2005, Astron. Astrophys., 436, 1103.

Dauphin, C., Vilmer, N., Anastasiadis, A.: 2007, Astron. Astrophys., 468, 273.

Deeg, H.J., Borovsky, J.E., Duric, N.: 1991, Phys. Fluids B, 3, 2660.

Dennis, B.R.: 1985, Solar Phys., 100, 465.

Efthymiopoulos, C., Gontikakis, C., Anastasiadis, A.: 2005, Astron. Astrophys., 443, 663.

Fletcher, L., Petkaki, P.: 1997, Solar Phys., 172, 267.

Gontikakis, C., Efthymiopoulos, C., Anastasiadis, A.: 2006, Mon. Not. Roy. Astron. Soc., $\mathbf{3 6 8}, 293$.

Gontikakis, C., Anastasiadis, A., Efthymiopoulos, C.: 2007, Mon. Not. Roy. Astron. Soc., $\mathbf{3 7 8}, 1019$.

Hamilton, B. McClements, K.G., Fletcher, L., Thyagaraja, A.: 2003, Solar Phys., 214, 339.

Hamilton, B., Fletcher, L., McClements, K.G., Thyagaraja, A.: 2005, Astrophys. J., 625, 496.

Hannah, I.G., Fletcher, L.: 2006, Solar Phys., 236, 59. 
Heerikhuisen, J., Litvinenko, Y.E., Craig, I.J.D.: 2002, Astrophys. J., 566, 512.

Hughes, D., Paczuski, M., Dendy, R.O., Helander, P., McClements, K.G.: 2003, Phys. Rev. Let., 90, 13, 131101.

Isliker, H., Anastasiadis, A., Vlahos, L.: 2001 Astron. Astrophys., 377, 1068.

Kliem, B.: 1994, Astrophys. J. Suppl., 90, 719.

Krucker, S., Benz, A.O.: 1998, Astrophys. J., 501, L213.

Litvinenko, Y.E.: 1996, Astrophys. J., 462, 997.

Litvinenko, Y.E., Somov, B.: 1993, Solar Phys., 146, 127

Martens, P.: 1988, Astrophys. J., 330, 131.

Martens, P., Young, A.: 1990, Astron. Astrophys. Suppl., 73, 333

Mercier, C., Trottet, G.: 1997 Astrophys. J., 474, L65.

Miller, J. A., Cargill, P.J., Emslie, A.G., Holman, G.D., Dennis B.R., LaRosa, T.N., et al.: 1997, J. Geophys. Res., 102, 14631

Mori, K.I., Sakai, J.I., Zhao, J.: 1998, Astrophys. J., 494, 430.

Moses, R.W., Finn, J.M., Ling, K.M.: 1993, J. Geophys. Res., 98, 4013.

Onofri, M., Isliker, H., Vlahos, L.: 2006, Phys. Rev. Let., 96, 1102.

Parker, E.N.: 1988, Astrophys. J., 330, 474

Petkaki, P., MacKinnon, A.L.: 1997, Solar Phys., 172, 279.

Petkaki, P., MacKinnon, A.L.: 2007, Astron. Astrophys., 472, 623.

Saint-Hilaire, P., Benz, A.O.: 2002, Solar Phys., 210, 287.

Speiser, T.W.: 1965, J. Geophys. Res., 70, 4219.

Tajima, T., Sakai, J., Nakajima, H., Kosugi, T., Brunel, F., Kundu, M.R.: 1987, Astrophys. J., 321, 1031

Vekstein, G.E., Browning, P.K.: 1997, Phys. Plasmas, 42261.

Vlahos, L., Isliker, H., Lepreti, F., 2004, Astrophys. J., 608, 540.

Wood, P., Neukirch, T.: 2005, Solar Phys., 226, 73.

Zharkova, V., Gordovskyy, M.: 2004, Astrophys. J., 604, 884.

Zharkova, V., Gordovskyy, M.: 2005, Mon. Not. Roy. Astron. Soc., 356, 1107. 
anastasi.tex; 7/11/2018; 8:21; p.18 